\title{
Computing Optimal Taxes in Atomic Congestion Games
}

\author{
Rahul Chandan \\ University of California, Santa Barbara \\ rchandan@ucsb.edu \\ Bryce L. Ferguson \\ University of California, Santa Barbara \\ blferguson@ece.ucsb.edu
}

\author{
Dario Paccagnan \\ University of California, Santa Barbara \\ dariop@ucsb.edu \\ Jason R. Marden \\ University of California, Santa Barbara \\ jrmarden@ece.ucsb.edu
}

\section{CCS CONCEPTS}

- Theory of computation $\rightarrow$ Algorithmic game theory; Network games; Algorithmic mechanism design.

When the performance of a system is dictated by the behaviour of its users, self-interested choices can result in sub-optimal system operation, as is the case in road traffic networks. The inefficiency resulting from such selfish behaviour is commonly measured by the ratio between the emergent worst-case system cost and the minimum system cost, termed price-of-anarchy. As the degree of inefficiency can be significant even for relatively simple systems (e.g., affine congestion games), researchers have proposed a variety of approaches to align the emergent selfish behaviour with the desired system objective. A well-studied and promising method is that of altering users' perceived costs by means of taxes.

With this same objective in mind, in this work, we investigate how to design optimal taxation mechanisms, i.e. we study how to design taxation mechanisms that minimize the system's inefficiency, relative to the class of atomic congestion games. In an atomic congestion game, a finite number of users choose from a subset of available edges. The congestion on each resource is a function of the number of users sharing it. Agents are selfish and aim at minimizing their individual experienced congestion, so that the emergent behaviour is well-captured by the notion of Nash equilibrium, or generalizations thereof (e.g., coarse-correlated equilibrium).

The need for taxes has been motivated by classic results demonstrating significant differences between equilibrium and optimal system performance $[1,2]$. In the absence of any taxation mechanism, selfish behaviour can result in a system cost as high as 2.5 times the optimal cost, relative to the class of atomic affine congestion games [3]. Even greater inefficiencies arise when higher-order polynomial latency functions are introduced.

There has been significant research in computing taxes that minimize the price-of-anarchy for a specific game instance. We term these taxes network-aware, based on the fact that they are computed on an instance-by-instance basis utilizing knowledge of the network structure. While such taxes provide a quantifiable inefficiency reduction, they require leveraging complete network and user information - a rather unrealistic scenario. In addition, the

Permission to make digital or hard copies of part or all of this work for personal or classroom use is granted without fee provided that copies are not made or distributed for profit or commercial advantage and that copies bear this notice and the full citation on the first page. Copyrights for third-party components of this work must be honored

For all other uses, contact the owner/author(s)

NetEcon'19, fune 28, 2019, Phoenix, AZ, USA

(c) 2019 Copyright held by the owner/author(s).

ACM ISBN 978-1-4503-6837-7/19/06.

https://doi.org/10.1145/3338506.3340239 resulting taxation mechanisms must be recomputed for any change in the network topology, which is problematic as the methods proposed have complexity growing exponentially with the number of users and the size of the network.

From the perspective of a system designer, a taxation mechanism that assigns a tolling function to each edge relative to the local latency function, and independent of the full network structure and user information, is far easier to implement and maintain. These taxation mechanisms - often termed network-agnostic - provide a degree of scalability and robustness to changes and errors that the alternative network-aware tolls lack. However, it has proven difficult to provide tight price-of-anarchy bounds for a given taxation mechanism, as one must consider its performance in each and every game instance of the class considered. Further and more fundamentally, there is currently no means to design an optimal network-agnostic tax or a discernible structure of such a mechanism. It is in this context that our work originates.

Contributions. The goal of this work is to quantify and optimize the price-of-anarchy resulting from network-agnostic taxation mechanisms, over the class of atomic congestion games. Our main contributions are as follows:

(1) We introduce a method for computing tight price-of-anarchy bounds, in any network-agnostic taxation mechanism, including, but not limited to, the discretized Pigouvian taxes.

(2) We design network-agnostic optimal taxation mechanisms that apply to congestion games with any form of positive latency function as the solution to a tractable linear program.

(3) We compute the price-of-anarchy under an optimal networkagnostic taxation mechanism, and compare it to the networkaware results in the literature for games with polynomial latency functions, though our formulation applies to any set of positive latencies.

In addition, we observe in this work that full network knowledge provides only minor gains in worst-case efficiency guarantees when optimal tolls are used, specifically in the case of congestion games with polynomial latency functions.

\section{ACKNOWLEDGMENTS}

This work is supported by ONR grant \#N00014-17-1-2060, NSF grant \#ECCS-1638214, and SNSF grant \#P2EZP2_181618.

\section{REFERENCES}

[1] Arthur Pigou. The economics of welfare. Routledge, 1920.

[2] Tim Roughgarden. How Bad Is Selfish Routing? f. ACM, 2002.

[3] George Christodoulou and Elias Koutsoupias. The price of anarchy of finite congestion games. ACM, 2005. 